Revue des patrimoines

$41 \mid 2019$

Patrimoines gastronomiques. Définitions, typologies et enjeux de conservation

\title{
Démarches participatives : fondements et pratiques actuelles dans les institutions muséales
}

Marie-Dominique Dubois

\section{(2) OpenEdition}

Journals

Édition électronique

URL : http://journals.openedition.org/insitu/27176

DOI : $10.4000 /$ insitu. 27176

ISSN : 1630-7305

Éditeur

Ministère de la Culture

Référence électronique

Marie-Dominique Dubois, « Démarches participatives : fondements et pratiques actuelles dans les institutions muséales », In Situ [En ligne], 41 | 2019, mis en ligne le 06 janvier 2020, consulté le 10 décembre 2020. URL : http://journals.openedition.org/insitu/27176 ; DOI : https://doi.org/10.4000/ insitu. 27176

Ce document a été généré automatiquement le 10 décembre 2020.

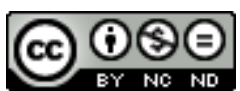

In Situ Revues des patrimoines est mis à disposition selon les termes de la licence Creative Commons Attribution - Pas d'Utilisation Commerciale - Pas de Modification 4.0 International. 


\title{
Démarches participatives : fondements et pratiques actuelles dans les institutions muséales
}

\author{
Marie-Dominique Dubois
}

1 En septembre 2019 s'est tenue à Kyoto (Japon) l'Assemblée générale extraordinaire de l'ICOM-Conseil international des musées afin d'adopter une nouvelle définition du musée. Le vote ayant été reporté, les discussions réunissant les membres du Conseil international des musées se poursuivent, au regard notamment des réflexions soumises par le comité permanent pour la Définition du musée, perspectives et potentiels (MDPP).

2 S'il est évidemment question de donner un nouveau cadre aux institutions muséales afin que celles-ci puissent pleinement jouer leur rôle social et sociétal dans un monde en profonde mutation, des tables rondes organisées en amont par le comité MDPP et réunissant des professionnels des musées ont rappelé la nécessité de tendre vers « des méthodes plus inclusives, ouvertes à la participation et à l'implication des communautés ${ }^{1} »$.

Qu'ils soient en phase d'expérimentation ou déjà bien rodés à ces pratiques, les musées - toutes catégories confondues - placent la participation des publics au cœur de leurs préoccupations. Un $\mathrm{Fab} \mathrm{Lab}^{2}$, c'est-à-dire un laboratoire de fabrication partagée en cours d'étude pour l'un, une collecte d'objets auprès des publics pour l'autre ou bien une exposition conçue par de jeunes adolescents... Les projets participatifs sont multiples et bel et bien dans l'air du temps. Reste que loin d'être un effet de mode, la participation constitue un moyen de répondre à l'égalité d'accès à la culture, de reconnaître les droits culturels ${ }^{3}$, d'être au service du développement de la société, "d'être utile et de retrouver le chemin de la légitimité4."

4 Mais quel sens donner à participation? Ce qui peut venir d'abord à l'esprit est proche de la médiation culturelle ou encore d'une visite immersive ou interactive à l'aide de supports numériques... Notre approche, reposant sur une recherche de terrain et d'étude des démarches participatives mises en œuvre par les musées, dans le cadre 
d'une thèse professionnelle, nous conduit à nous interroger sur la notion et la définition d'une démarche participative, à en découvrir ses fondements et ses caractéristiques, qui la distinguent de fait d'une action de médiation culturelle, même si celle-ci en constitue un premier stade. C'est par une analyse historique que nous amorcerons notre propos avant d'en observer des illustrations concrètes contemporaines, en France et en Europe.

\section{Émergence et fondements des démarches participatives}

5 Si la tendance peut paraître assez récente, notamment dans les musées de beaux-arts, la participation est l'ADN des écomusées qui apparaissent, en France, à la fin des années 1960. Certains ont été créés au sein d'un parc naturel régional (PNR), à l'instar de l'écomusée de Niou à Ouessant, construit en 1968 dans le parc naturel régional d'Armorique, ou encore, un an plus tard, l'écomusée de la Grande Lande à Marquèze, dans le parc naturel régional des Landes de Gascogne.

Un écomusée est une institution culturelle qui assure, de manière permanente, sur un territoire donné, avec la participation de la population, les fonctions de recherche, conservation, présentation, mise en valeur d'un ensemble de biens naturels et culturels représentatifs d'un milieu et des modes de vie qui s'y succèdent ${ }^{5}$. Les habitants, contribuant activement et bénévolement, jouent différents rôles. Un jour, ils assurent une mission de collecte d'objets, un autre, ils font office de médiateurs auprès du jeune public, une autre fois encore, ils interviennent en tant que membres du comité des usagers afin de proposer entre autres un programme d'action pour l'écomusée. En bref, aux côtés de l'équipe scientifique ayant des compétences spécifiques, les habitants jouent un rôle déterminant. Ces derniers agissent tels des professionnels et les connaissances qu'ils ont d'eux-mêmes et de leur territoire ont autant de valeur que celles des scientifiques.

7 À l'écomusée du Val de Bièvre, situé à Fresnes (Val-de-Marne), en Île-de-France, les actions participatives sont considérées comme un moyen de compléter la connaissance du territoire et d'enrichir les collections. Celles-ci font émerger des sujets qui ne sont pas souvent traités par les musées mais font néanmoins partie de la réalité et de la diversité du territoire ${ }^{6}$.

Dans une démarche participative, le récit est écrit ainsi à plusieurs voix. Il s'agit d'une élaboration commune, d'une nouvelle forme de dialogue où le non-professionnel - le non-sachant - tend à se situer quasiment sur un pied d'égalité avec le professionnel - le sachant -. La démarche participative revient à reconnaître le potentiel et la compétence de chacun. La participation se révèle dans un processus de coconstruction.

9 C'est ainsi qu'il convient de différencier démarche participative et médiation culturelle ${ }^{7}$. En effet, la médiation suppose que les contenus et les résultats d'une action ponctuelle soient définis en amont par les équipes du musée. Par exemple, un atelier créatif à la suite de la visite d'une exposition d'art pourra consister à s'initier à une technique observée au cours de l'exposition et aboutir à une création. De même, au cours d'une visite, si le professionnel, qu'il soit conférencier ou médiateur culturel, 
cherche à favoriser auprès des visiteurs le partage d'expériences et la diversité d'expression, la visite suit un fil conducteur.

10 À l'inverse, une démarche participative ne suppose d'être définie ni dans son contenu ni dans son résultat. Il convient de laisser une place à l'imprévu et au lâcher prise. Quant au professionnel, il endosse ici un rôle d'accompagnateur du projet et d'interface - en général sur une temporalité longue - entre les différents acteurs du projet (travailleurs sociaux, éducatifs, institutionnels, habitants...). Des ajustements et des ouvertures entre l'institution culturelle et les acteurs pré-cités sont nécessaires tout au long du processus. In fine, l'important n'est pas tant le résultat que la méthode d'élaboration du projet. Tout projet naît de la rencontre entre plusieurs partenaires qui vont favoriser la création d'un espace social propice à l'échange.

11 Dans les écomusées, l'importante diminution du nombre de bénévoles a amené à repenser les modes de participation des habitants afin que celle-ci reste ancrée dans la conduite du projet scientifique et culturel de l'établissement. Une ambition partagée plus largement par les musées de société, appellation qui fait référence à tout musée se caractérisant par un rôle de conservatoire, d'étude et de valorisation des collections composées d'objets ou de documents témoins de l'évolution de l'homme ${ }^{8}$. Au sein de ces catégories d'établissements culturels, quels sont les projets participatifs? En quoi consistent-ils ? Et jusqu'à quel niveau les publics sont-ils impliqués?

\section{Exemples de démarches participatives}

12 En fonction de l'institution muséale et de son degré d'ouverture à la démarche participative, celle-ci se déploie à différents stades. D'une création commune d'un parcours de visite à une participation à la médiation culturelle jusqu'au cocommissariat d'exposition, en passant par une co-production de contenus exposés. Loin d'être exhaustif, il s'agit de se pencher essentiellement sur des bonnes pratiques dont certaines ont été pionnières en France et d'autres plus répandues.

\section{Co-création d'expériences de visite}

13 Avant de rentrer dans la phase de production d'une offre culturelle, dans le cadre de la rénovation de son parcours permanent, le musée national de la Marine, situé dans le $16^{\mathrm{e}}$ arrondissement de Paris, a souhaité co-créer l'offre pour les publics scolaires. Pour cela, le musée a réuni les principaux intéressés en recevant, en février 2019, une quarantaine d'élèves de niveau collège et lycée. Durant une journée, sous la forme d'un marathon créatif ${ }^{9}$, les élèves, accompagnés par les équipes du musée et les partenaires pédagogiques ${ }^{10}$, ont imaginé des propositions de parcours, des dispositifs de médiation et des supports de visite sur des thématiques variées : « Des histoires de marins », «Un musée pour tous », « De l'aventure » ...

14 Le retour d'expérience des équipes du musée national de la Marine a été très positif, non seulement sur le niveau d'implication des adolescents mais aussi sur leurs propositions, très qualitatives. D'une manière très libre et spontanée, sans connaissance préalable du musée ou des collections, les élèves ont réalisé des vidéos, des maquettes en carton ou des murs illustrés de post-it avec des mots clés. L'ensemble des données collectées sera analysé attentivement pour que le musée nourrisse une réflexion sur le renouvellement de son discours auprès des publics scolaires, pour être 
en mesure de valoriser tel ou tel outil, de justifier ou de modifier une approche. L'institution muséale montre aussi sa volonté de se reconnecter avec ce public et de créer un lien avec les jeunes visiteurs. Dans un avenir proche, l'établissement envisage de poursuivre sa démarche en invitant quelques élèves ayant participé au marathon créatif à venir tester et évaluer les outils conçus a posteriori par les professionnels.

Pratiquée dans les écomusées et courante dans les musées de société, la collecte de connaissances, d'objets ou de témoignages auprès des publics est une autre illustration fréquemment observée de démarche participative. Dans le cadre d'une exposition, permanente ou temporaire, il s'agit de collecter des histoires individuelles pouvant résonner collectivement et de les replacer dans un contexte contemporain. Incontestablement, recueillir la mémoire collective donne à une thématique un point de vue plus concret, plus émotionnel, plus affectif. Par cette démarche, l'objectif poursuivi est entre autres de donner la parole au public; sans s'affranchir d'une expertise scientifique, la collecte permet d'enrichir le contenu exposé et de montrer une diversité de points de vue. Fortes de l'attachement collectif au patrimoine, les démarches de collecte trouvent fréquemment leur public.

\section{Collectes : objets, témoignages et savoirs}

Au musée départemental de l'Arles antique, dans le cadre de l'exposition "J'aimerais tant voir Syracuse ", le musée a invité le public à ouvrir les albums de famille pour une collecte intitulée "L'Antique e(st) nous ». Au total, mille deux cents clichés montrant des personnes aux côtés d'un monument antique ou tout vestige de site archéologique de la Narbonnaise et du pourtour méditerranéen ont été collectés. Cette opération a montré l'attachement collectif au patrimoine archéologique.

Un constat partagé par le Laténium, parc et musée d'Archéologie de Neuchâtel (Suisse), à l'occasion d'une collecte de photographies vernaculaires sur lesquelles les personnes sont photographiées devant des éléments et sites du patrimoine archéologique suisse. Celles-ci ont été collectées pour une exposition participative temporaire, "Émotions patrimoniales $»^{11}$. Disponible à l'inattendu et ouvert à ce qui « fait patrimoine » auprès du grand public, le musée n'a pas cherché à définir scientifiquement le patrimoine archéologique mais à accueillir les différentes manières de le penser. La collecte a réuni cinq cents photographies retraçant plusieurs périodes historiques.

Autre exemple de collecte réussie, au musée portuaire de Dunkerque, dans le cadre de l'exposition "Oily Days ", traitant de l'industrie pétrolière locale de 1861 à 2010, le public a été invité à apporter des jouets (jouets de la marque Esso, station essence Playmobil, voitures modèles réduits des années 1960...), des accessoires et du mobilier (seau à glaçons en forme de pomme, tabouret orange en forme de tam-tam...). L'appel à collecte a été lancé sur les réseaux sociaux puis relayé par un réseau d'associations et de partenaires, ces derniers maillons étant indispensables afin de toucher le maximum de personnes.

19 C'est ainsi que pour l'exposition " Face au génocide, du Cambodge à l'Isère ", en 2008, les équipes du musée de la Résistance et de la Déportation de l'Isère, à Grenoble, ont travaillé étroitement avec des associations et recueilli des témoignages auprès d'une dizaine de victimes du génocide. De même, à l'écomusée du Pays de Rennes, une prochaine exposition consacrée à l'immigration sera construite à partir de récits de vie collectés par l'intermédiaire d'associations. 
20 Faire collaborer les habitants à la définition du patrimoine est aussi une démarche fréquemment mise en œuvre par l'écomusée du Val de Bièvre. Pour la dernière collecte en date, dans le cadre de l'exposition " Objets privés, objets partagés ", ayant trait aux aspects de la vie en banlieue parisienne, les équipes de l'écomusée se sont inspirées du concept de la collecte écomuséale ${ }^{12}$ développée au Québec, à l'écomusée du Fier Monde. Pour statuer sur la valeur patrimoniale des objets collectés, un comité de pilotage constitué à parts égales d'habitants, d'universitaires et de professionnels des musées a été créé. Certains des objets non retenus ont été exposés dans un espace dédié à la fin $\mathrm{du}$ parcours de l'exposition, indiquant les raisons pour lesquelles ils n'avaient pas suscité l'adhésion. Créer des espaces dédiés aux résultats d'une collecte permet à la fois de valoriser la contribution de chacun et d'encourager les visiteurs à poursuivre la démarche. C'est ainsi qu'au Laténium, une table tactile installée à l'entrée de l'exposition temporaire précédemment citée, "Émotions patrimoniales", montre l'ensemble des photographies collectées et légendées.

21 Parallèlement à une collecte aboutissant à une exposition, la démarche participative s'observe aussi à travers la collecte de savoirs et de connaissances. Par exemple, au Muséum d'histoire naturelle à Paris, dans le cadre du programme de sciences participatives ${ }^{13}$ Vigie-Nature ${ }^{14}$ regroupant huit observatoires participatifs, le grand public - quel que soit son niveau de connaissances de la faune et de la flore - peut contribuer à la recherche en découvrant la biodiversité environnante. Chaque année, environ 15000 contributeurs - 13000 individuels, 400 classes, en majorité de niveau collège, et 2000 naturalistes - participent et envoient leurs observations dûment remplies à l'aide de protocoles et de fiches de terrain co-construits par le Muséum et les associations spécialisées. Les observations du grand public portent sur les oiseaux à mangeoire, les papillons, les bourdons, les escargots... Des données larges à la fois dans l'espace et dans le temps, essentielles pour les chercheurs. Ces derniers les exploitent afin de mesurer le déclin de la biodiversité et la façon dont celle-ci évolue face au réchauffement climatique ou à l'intensification de l'agriculture. Les participants obtiennent des retours concrets sur l'utilisation des données transmises.

La co-construction de savoirs et de connaissances est aussi expérimentée depuis 2017 au musée de Bretagne, musée de société et d'histoire de la ville de Rennes, à travers la plateforme en ligne " Des collections en partage », créée en partenariat avec l'écomusée du Pays de Rennes. La plateforme propose à l'internaute d'identifier un lieu, une personne ou l'usage d'un objet parmi les milliers d'objets d'art et de documents numérisés, un certain nombre d'images étant non identifiées. Des ajouts, des informations très précises et des messages sont régulièrement transmis par les internautes qui contribuent ainsi à l'élaboration d'une matière scientifique.

Il est particulièrement intéressant de constater que ces démarches participatives s'étendent aussi à la conception d'une exposition ou au contenu d'une programmation culturelle. Expérimentés dans de nombreuses institutions muséales, y compris dans des musées de beaux-arts et des établissements patrimoniaux, des projets participatifs sont renouvelés chaque année, forts de leur succès.

\section{Co-conception d'exposition et accrochages participatifs}

24 Au musée des Civilisations de l'Europe et de la Méditerranée (MUCEM), à Marseille, vingt-quatre collégiens de troisième ont été invités à imaginer une exposition, «Osez 
l'interdit" $(2019)^{15}$. Les élèves ont choisi des œuvres dans les réserves du musée pour imaginer une muséographie autour du thème de l'interdit. Ils ont rencontré des professionnels et une équipe pédagogique a été mobilisée.

Citons également l'exemple du musée Sainte-Croix, musée d'art et d'archéologie de Poitiers, où des adolescents ont réalisé une exposition intitulée «Un autre regard, les jeunes traînent aussi au musée " (2015-2016). Après avoir sélectionné une douzaine d'œuvres dans les importantes réserves de l'établissement, les jeunes gens ont conçu leur propre accrochage d'œuvres en travaillant main dans la main avec les différents départements du musée. Cette exposition a été le fruit d'un partenariat tissé avec Le Local, un centre socioculturel de la ville de Poitiers.

Autre dispositif, inauguré en 2016 par la Réunion des musées métropolitains (RMM) Rouen-Normandie et reconduit depuis chaque année, l'opération «La Chambre des visiteurs " permet au public de choisir parmi une sélection d'œuvres conservées dans les réserves des huit musées de la RMM celles qui seront exposées quatre à cinq mois dans les salles d'exposition. S'inspirant d'expositions participatives développées outreAtlantique et en Europe, le dispositif fut une première en France. Le succès est au rendez-vous : pour la première édition, 17000 votes ont été comptabilisés. Grâce à ce dispositif, le public peut apporter son regard sans que cela ne remette en cause l'expertise de l'institution, et parfois même, participer à l'écriture de cartels de certaines œuvres avec l'équipe scientifique dans le cadre d'ateliers d'écriture.

\section{Co-création de projets culturels et participation à la médiation culturelle}

27 Au Centre Georges-Pompidou (Paris), encadré par la direction des Publics, le collectif Art Session ${ }^{16}$ recrute chaque année une vingtaine de jeunes bénévoles, âgés de 18 à 25 ans. Ces derniers contribuent activement à alimenter et à donner naissance à des projets culturels par et pour les jeunes dans le cadre de la médiation culturelle ou dans les moyens d'accéder aux ressources de l'institution. Les jeunes bénévoles contribuent au bon déroulement de la programmation culturelle. Par exemple, dans le cadre du festival pluridisciplinaire Hors Pistes, avec pour thème de la 14 édition, la Lune (2019), les jeunes bénévoles participent à la médiation et à des ateliers créatifs. Ils sont aussi amenés à rencontrer des artistes professionnels, à visiter des expositions et à partager leur expérience auprès des équipes à propos de la fluidité du parcours ou de la lisibilité des cartels dans les salles d'exposition.

La dimension participative se remarque en effet jusque dans les salles d'exposition, face au public. Une fois par an, la Réunion des musées nationaux-Grand Palais organise l'événement "Tous au Grand Palais », une journée au cours de laquelle des lycéens et des étudiants deviennent médiateurs culturels dans les salles d'une exposition temporaire du Grand Palais. Les jeunes gens sont inscrits dans des établissements scolaires situés en zone d'éducation prioritaire (ZEP) et faisant partie du programme d'égalité des chances développé par la Fondation Paris Dauphine. Pour préparer cette journée, les participants ont étudié des commentaires d'œuvres d'art, choisies en amont, et extraits du catalogue d'exposition. Mais leur discours, loin d'être uniforme, laisse place à une liberté d'expression et à un rapport spontané avec les publics. Les résultats très positifs se situent très souvent au-delà des attentes. 
o-construit avec les partenaires pédagogiques, ce projet est porté par les jeunes participants volontaires. L'envie est le premier facteur de réussite d'un tel projet ${ }^{17}$. Forts d'un enrichissement personnel et fiers d'avoir relevé le défi de prendre la parole en public, un certain nombre de participants renouvellent l'expérience et deviennent en quelque sorte des mentors pour les plus novices. "Cette expérience les amène aussi à prendre conscience de leurs compétences, de ce qu'ils aiment faire ou ne pas faire ${ }^{18}$. » Ici, la démarche peut amener à changer le point de vue que l'on porte sur soi-même et peut-être sur l'autre ; le public peut quant à lui être amené à poser un autre regard sur ces jeunes issus de quartiers défavorisés.

\section{Conclusion}

La diversité et l'inventivité des projets participatifs conduits par des professionnels exigeants prouvent qu'il s'agit bien d'une réalité tangible avec des conséquences sur l'institution culturelle, sa posture, sa muséographie, ses espaces d'accueil du public. Les exemples de pratiques précédemment cités doivent être complétés par ceux de projets culturels menés entre autres dans le cadre du Parcours d'éducation artistique et culturelle (PEAC) et fondés sur une participation active du jeune public.

Plus largement, il est question pour le musée d'être une maison commune, perméable à la société civile et ouvert à la création contemporaine, que celle-ci soit réalisée par des artistes professionnels ou par des amateurs. Une profonde réflexion sur la démarche participative est engagée pour aujourd'hui et pour demain. Cette démarche constitue un levier susceptible de répondre aux enjeux contemporains car elle peut être l'occasion de faire tomber des barrières et offre à tout un chacun une parenthèse artistique et culturelle.

\section{NOTES}

1. - Rapport pour le Conseil d'administration de l'ICOM, décembre 2018. Voir sur le site : https:// icom.museum/wp-content/uploads/2019/01/MDPP-report-and-recommendations-adopted-bythe-ICOM-EB-December-2018_FR.pdf [consulté le 06/01/2020].

2. - Un Fab Lab (contraction de l'anglais fabrication laboratory, « laboratoire de fabrication ») est un lieu ouvert au public où il est mis à sa disposition toutes sortes d'outils, notamment des machines-outils pilotées par ordinateur, pour la conception et la réalisation d'objets. Voir le site : http://carrefour-numerique.cite-sciences.fr/fablab/wiki/doku.php?id=charte [consulté le 06/01/2020].

3. - Les droits culturels, parties intégrantes des droits de l'homme, désignent les droits, libertés et responsabilités pour une personne, seule ou en commun, de choisir et d'exprimer son identité, ce qui suppose la possibilité d'accéder, de pratiquer et de contribuer à une diversité de références et ressources culturelles de qualité, qui sont nécessaires à son processus d'identification, de communication et de création. Ils constituent les capacités de lier les personnes à une diversité de savoirs portés par d'autres personnes, des institutions ou déposés dans des objets. Autrement dit, ils rendent chacun capable de puiser dans les œuvres comme en autant de références qui sont 
des ressources indispensables à son développement. Voir la Déclaration de Fribourg sur les droits culturels : https://droitsculturels.org/wp-content/uploads/2012/07/DeclarationFribourg.pdf [consulté le 06/01/2020].

4. - Extrait d'un entretien téléphonique le 24 janvier 2019 avec M. Sylvain AMIC, directeur de la Réunion des musées métropolitains (RMM) - Rouen Normandie (DUBOIS, Marie-Dominique. La Participation des publics : de la démarche participative développée par les écomusées et les musées de société à celle des musées de beaux-arts. Regard et analyses. Mastère spécialisé en Management des entreprises culturelles et des industries créatives, Burgundy School of Business, 2019, p. 147).

5. - Article 1 de la Charte des écomusées, instruction du 4 mars 1981, du ministre de la Culture et de la Communication. Voir le site: http://fems.asso.fr/wp-content/uploads/2018/03/Charte\%C3\%A9comus\%C3\%A9es.pdf [consulté le 06/01/2020].

6. - Voir le site : http://ecomusee.agglo-valdebievre.fr/page/les-actions-participatives [consulté le 06/01/2020].

7. - La médiation culturelle désigne toute une gamme d'interventions établissant des ponts entre ce qui est exposé (le voir) et les significations que ces objets et sites peuvent revêtir (le savoir) (DESVALLÉES, André et MAIRESSE, François. Dictionnaire encyclopédique de muséologie. Paris: Armand Colin, 2011).

8. - Les musées de société peuvent être des musées d'histoire, de science et de technologie, d'art et d'archéologie ou encore de sciences naturelles pour autant que leur questionnement tourne autour de l'homme et de la vie en société (DROUGUET, Noémie. Le Musée de société. De l'exposition de folklore aux enjeux contemporains. Paris : Armand Colin, 2015).

9. - Voir le site: http://www.musee marine.fr/blog/musee_marine_renovation/2019/04/26/ revisithon-le-musee-national-de-la-marine-un-marathon-creatif-et-pedagogique/ [consulté le 06/01/2020].

10. - Le projet a été accompagné par le réseau Canopé île-de-France, voir le site: https:// www.reseau-canope.fr/ [consulté le 06/01/2020].

11. - Voir le site : http://latenium.ch/expo/exposition-temporaire/ [consulté le 06/01/2020].

12. - La collecte écomuséale se compose d'éléments matériels et immatériels qui ont une signification particulière pour la compréhension du territoire. Il peut s'agir de bâtiments, de sites, d'infrastructures... Ces éléments sont désignés mais ne deviennent pas la propriété de l'écomusée. L'originalité de la démarche repose sur la contribution des résidents du quartier. Ils participent à l'identification des éléments et contribuent à leur mise en valeur en s'impliquant au sein d'un comité des parrains et des marraines du patrimoine. La collection écomuséale devient ainsi un outil de développement local. Elle permet aux citoyens de s'approprier leur histoire, leur patrimoine et renforce le sentiment d'appartenance au quartier.

13. - Selon François Houllier, biologiste français, alors directeur de l'INRA, «les "sciences participatives" sont des formes de production de connaissances scientifiques auxquelles des acteurs non-scientifiques-professionnels - qu'il s'agisse d'individus ou de groupes - participent de façon active et délibérée ». Définition à retrouver dans le rapport remis au ministère de l'Éducation nationale en février 2016: HOULLIER, François (dir.). Les Sciences participatives en France. État des lieux, bonnes pratiques et recommandations. Voir sur le site: Mission Sciences participatives - Rapport [consulté le 06/01/2020].

14. - Programme de sciences participatives Vigie-Nature, voir le site: https://www.mnhn.fr/fr/ participez/contribuez-sciences-participatives/observatoires-vigie-nature [consulté le 06/01/2020].

15. - Descriptif de l'exposition «Osez l'interdit, les jeunes font leur musée ». Voir le site : http:// www.mucem.org/programme/exposition-et-temps-forts/osez-linterdit [consulté le 22/05/2019].

16. - Descriptif du collectif Art Session. Voir le site: https://www.centrepompidou.fr/cpv/ resource/czzgLaq/rR5njdG [consulté le 22/05/2019]. 
17. - Extrait d'un entretien en face à face le 2 avril 2019 avec Mme Christine Perney, chargée de projets culturels « réseaux éducatifs » à la Réunion des musées nationaux-Grand Palais (DUBOIS, Marie-Dominique. La Participation des publics...Op. cit., p. 150).

18. - Ibid.

\section{RÉSUMÉS}

Dans les années 1970, les écomusées en France inscrivent la participation des habitants au cœur de leur mode de fonctionnement. Depuis, les processus participatifs initialement portés par un important mouvement bénévole ont évolué vers de nouveaux formats, formats qui répondent toutefois à des caractéristiques distinguant une démarche participative d'une action de médiation culturelle. Sa philosophie: faire interagir plusieurs acteurs de la société civile (travailleurs sociaux, éducatifs, habitants...) afin de co-construire un projet culturel. Nous revenons ici sur les fondements des démarches participatives et en retraçons le développement progressif, notamment dans les musées de beaux-arts, à l'appui d'exemples concrets illustrant des bonnes pratiques. Celles-ci présentent un degré plus ou moins élevé d'implication des publics, dans le temps ou dans l'action, mais toutes sont guidées par un objectif d'ouverture, de partage et de promotion de la diversité culturelle.

\section{INDEX}

Mots-clés : démarche participative, écomusée, participation des habitants, musée de société, collectes, témoignages, objets, co-construction, co-création d'expériences de visite, participation à la médiation culturelle, accrochages participatifs, droits culturels, éducation artistique et culturelle, musée de beaux-arts

\section{AUTEUR}

\section{MARIE-DOMINIQUE DUBOIS}

Diplômée en mastère spécialisé Management des entreprises culturelles et des industries créatives, Burgundy School of Business mariedominique.dubois@hotmail.com 